\title{
Effect of Kashima's Surgery in Bilateral Abductor Vocal Cord Palsy
}

\author{
Manju E Issac
}

\begin{abstract}
Introduction: Bilateral abductor palsy is treated by tracheostomy or any lateralization procedure. Kashima's surgery is a lateralization procedure introduced by Kashima in 1989.
\end{abstract}

Objective: To evaluate the effect of Kashima's surgery using subjective measures of breathing quality in patients with bilateral abductor palsy.

Materials and methods: This is a prospective study with 31 patients ( 3 men, 28 women) who underwent Kashima's surgery between January 2013 and December 2015 in the Laryngology department. Subjective evaluation was done by using dyspnea index score in the pre- and postoperative periods. Dyspnea index score is calculated based on answers given to a 7-point questionnaire and assessed by Likert scale, a self-assessment scale. For each point, the scores range from 0 to 3 , and the maximum score is 21 . Patients who already had tracheotomy were given a score of 14 . This score was calculated again using the same questionnaire in the pretracheostomy scenario.

Results: There is significant reduction in the dyspnea index score with a median reduction score of 9 and improvement of quality-of-life after Kashima's surgery.

Conclusion: Dyspnea index score is easy to administer. Considering the cost of treatment and high success rate of decannulation, it is obvious that Kashima's surgery is effective and affordable in bilateral abductor palsy.

Keywords: Dyspnea, Kashima's surgery, Vocal cord palsy.

How to cite this article: Issac ME. Effect of Kashima's Surgery in Bilateral Abductor Vocal Cord Palsy. Int J Phonosurg Laryngol 2017;7(1):10-12.

\section{Source of support: Nil}

Conflict of interest: None

\section{INTRODUCTION}

Evaluation and treatment of bilateral vocal fold paralysis carry significant challenges since the surgeon deals with the critical airway and the difficulty in selecting a treatment plan. In most of the acute airway emergencies,

\section{Associate Consultant}

Department of Laryngology, Kerala Institute of Medical Science Thiruvananthapuram, Kerala, India

Corresponding Author: Manju E Issac, Associate Consultant Department of Laryngology, Kerala Institute of Medical Science Thiruvananthapuram, Kerala, India, Phone: +919447238839 e-mail: manjujacob@hotmail.com the treatment offered is tracheostomy, which provides excellent airway as well as good voice. But, later on, most of the patients will prefer to avoid the burden of living with a tube in the neck. The stigma and the social disgrace of keeping a tube in the neck result in poor quality-of-life. So, the idea of improving the airway without tracheostomy has led to a number of surgical techniques. There were attempts to create more space at the level of the glottis or recreate the nerve function. The attempts to anastomose the cut nerve ending were made in 1920s, but later, it was abandoned due to lack of specificity of innervation. ${ }^{1}$

In 1916, Baker ${ }^{2}$ performed the first midline thyrotomy to attempt lateralization by removing cord and arytenoid in a 9-year-old boy. Jackson ${ }^{3}$ introduced simple cordectomy to improve the airway and later modified the procedure by removal of ventricle along with vocal cord. But, there was scarring, which led to stenosis. In 1936, Lore removed arytenoid cartilage for more lateralization of vocal cord. King ${ }^{4}$ introduced an external approach to lateralize the glottis. Anterior belly of omohyoid was attached to arytenoid cartilage to make more space posteriorly. Morrison ${ }^{5}$ added many modifications to this like disarticulation of arytenoid cartilage, division of interarytenoid muscle, and removal of arytenoid through a window created in the thyroid cartilage. In 1946, Woodman ${ }^{6}$ introduced the technique of external arytenoidectomy. This is the preferred approach in patients with difficult endoscopic exposure. Thornell, ${ }^{7}$ in 1948, introduced the first endoscopic arytenoidectomy to improve the airway. In 1989, Kashima introduced the most successful lateralization procedure, endoscopic laser posterior cordotomy, in which vocal fold is cut transversely just in front of the vocal process. A small portion of false cord is also removed. Tissue resection advances laterally until the inner perichondrium of thyroid and cricoid cartilage is reached. ${ }^{8}$

In our study, the effect of endoscopic laser posterior cordotomy or Kashima's surgery in bilateral vocal fold paralysis was evaluated. The improvement in quality-oflife in terms of improvement in dyspnea index score and successful decannulation was noted.

\section{MATERIALS AND METHODS}

A prospective study was conducted on 31 patients from the period January 2013 to December 2015. Dyspnea 
Effect of Kashima's Surgery in Bilateral Abductor Vocal Cord Palsy

index score was used to assess the effect of Kashima's surgery on bilateral vocal fold palsy. Only known cases of bilateral abductor palsy of more than 6 months duration were included in the study. Among the causes of bilateral vocal fold palsy, total thyroidectomy was the most common cause. Other causes were skull base surgery and idiopathic. In idiopathic cases, computed tomography scan from skull base to sternal angle was advised. Along with the diagnostic workup, a questionnaire based on subjective measurement of breathing quality was given to all the patients. Patients were asked to mark the appropriate score to the questions asked. We did not do a prophylactic tracheostomy in our cases. Kashima's surgery is done under general anesthesia. All laser-protective measures were taken during surgery like laser-resistant endotracheal tube. In tracheostomized cases, the laser flex tube is introduced through the stoma. Transverse cut is made just anterior to the vocal process. Tissue resection is carried out laterally until the inner perichondrium of cricoid and thyroid cartilage is reached. A portion of false cord is also cut to expose the surgical site. Mitomycin C is applied to reduce the chance of granulation formation (Fig. 1). Patients without tracheostomy were treated in the intensive care unit setup in the immediate postoperative period with antibiotics, analgesics, oxygen, and propped up position. Intravenous steroids were given in the absence of contraindication. Intravenous proton pump inhibitors are prophylactically used. Patients were discharged on 3rd postoperative day.

The most common problem encountered during surgery was bleeding, which was managed by applying laser or cautery. The dyspnea index scoring was done both preoperatively and postoperatively and the difference calculated.

Dyspnea index score is a subjective measure of breathing quality. It is based on answers given to seven

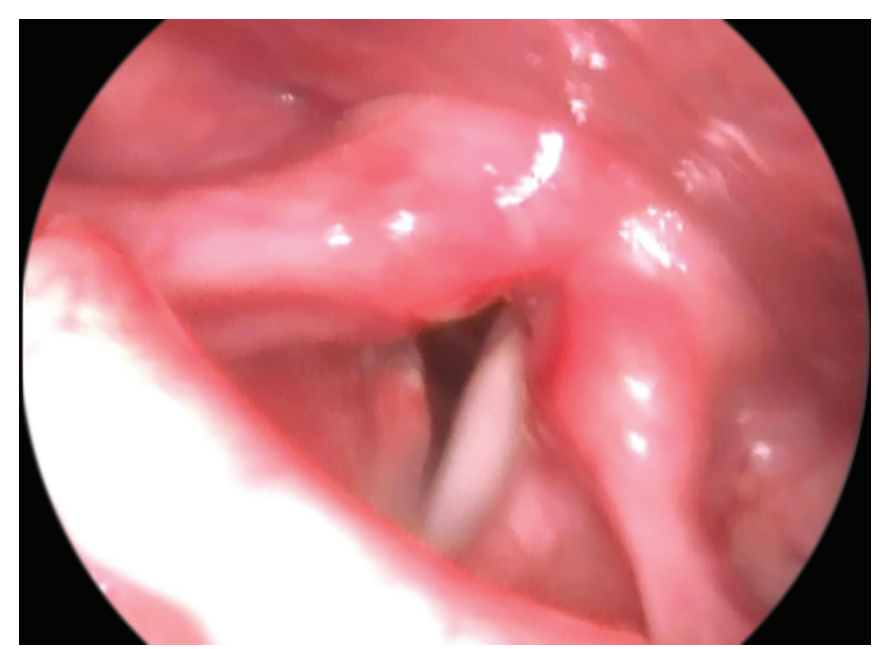

Fig. 1: Post-Kashima's surgery (4 weeks)
Table 1: Dyspnea index score

\begin{tabular}{lllll}
\hline Dyspnea at rest & 0 (nil) & 1 (mild) & 2 (moderate) & 3 (severe) \\
\hline $\begin{array}{l}\text { Dyspnea at light } \\
\text { work }\end{array}$ & 0 & 1 & 2 & 3 \\
$\begin{array}{l}\text { Dyspnea at } \\
\text { exertion }\end{array}$ & 0 & 1 & 2 & 3 \\
$\begin{array}{l}\text { Snoring, } \\
\text { disturbed sleep }\end{array}$ & 0 & 1 & 2 & 3 \\
$\begin{array}{l}\text { Weight loss } \\
\text { Dyspnea at URTI }\end{array}$ & 0 & 1 & 2 (nil) & 2 (yes) \\
Stridor & 0 (nil) & 1 (exertion) 2 (light work) & 3 (at rest) \\
\hline
\end{tabular}

URTI: Upper respiratory tract infection

questions on subjective measures of breathing quality. The scores range from 0 to 3 . The maximum score is 20 . Those who were already on tracheostomy were given a score of 14 since this was the most common score in those without tracheostomy. The questions were assessed with Likert scale, a subjective measurement of scoring system.

The questions with their scores are given in Table 1. The questions are based on whether the patients have shortness of breath during rest, light work, heavy work or exertion, or upper respiratory infection. In most of the cases, snoring was a major complaint. So, it was included in the questionnaire. Noisy breathing or stridor was included and scores given for its presence or absence during different ranges of exertion or rest. A high score was given for stridor which was present even at rest.

Weight loss was also given a score of 2 since it represents severe airway compromise.

Both preoperative and postoperative scoring were done and the difference calculated.

Since the outcome is score, this was analyzed using nonparametric tests, namely Wilcoxon's signed rank test, which is the nonparametric equivalent of paired t-test. There was significant reduction in the dyspnea index score postoperatively with an emerging median score of 9 .

\section{RESULTS}

This prospective study of 31 patients which consists of 28 females (90\%) and 3 males $(9.67 \%)$ revealed a significant reduction in dyspnea index score post Kashima's surgery. The age group ranges from 18 to 67 , with a mean age of 46.68 years. Out of 31 patients, 26 were postthyroidectomy cases $(83.87 \%)$ and 3 were idiopathic $(9 \%)$. There was a congenital case and one skull base surgery case $(6 \%)$. The mean preoperative score was 15.41 , with mode being 14 , and the mean postoperative score was 6.25 , with mode being 6.5. Difference in mean and mode preoperative and postoperative scores was 9.29 and 7 respectively. Since there were differences in scores, Wilcoxon signed rank test was applied. This is the nonparametric equivalent of paired t-test. The median reduction score was 9. Box and 


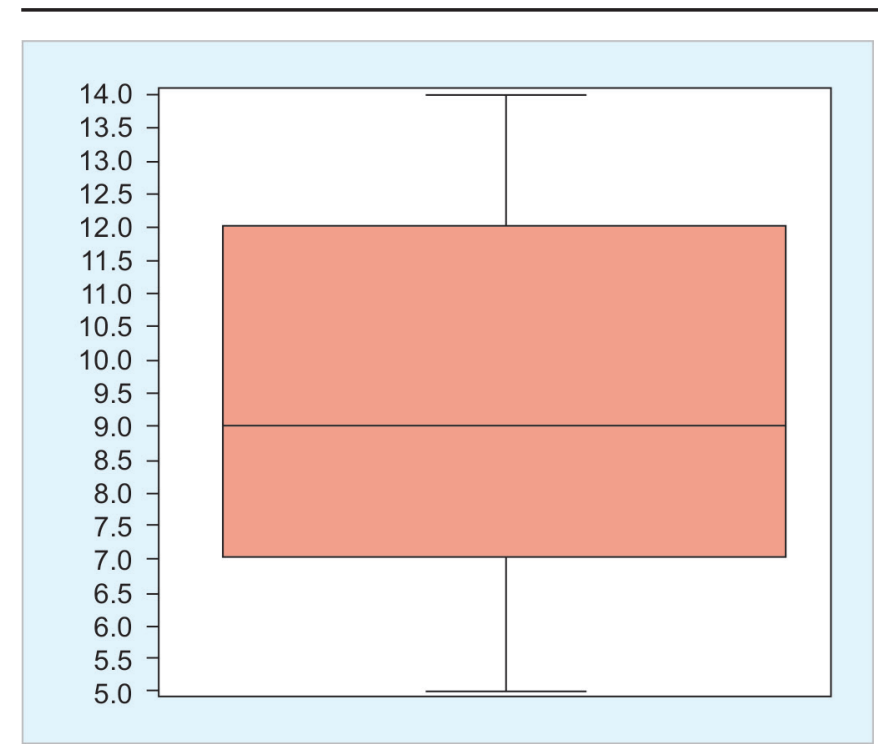

Graph 1: Box and whisker plot drawn to show the median reduction score of 9

Table 2: Median reduction score

\begin{tabular}{llll}
\hline Score & Prescore & Postscore & Difference \\
\hline Mean & 15.41 & 6.25 & 9.29 \\
Mode & 14 & 6.5 & 7 \\
p-value & & & 0.001 \\
\hline
\end{tabular}

whisker plot was drawn to show the median reduction score of 9 (Graph 1). The p-value in reduction is 0.001 , which is statistically significant (Table 2).

There were nine tracheostomized cases in the series and all were decannulated successfully after Kashima's surgery.

\section{DISCUSSION}

Aerodynamic, acoustic, and functional results of posterior transverse laser cordotomy for bilateral abductor vocal fold palsy were studied by Dursun and Gökcan. ${ }^{9}$ The modification of posterior cordotomy in bilateral abductor palsy was studied by John Segas et al. In their series, all patients had a temporary tracheostomy. ${ }^{10}$

The endoscopic laser posterior cordotomy or Kashima's surgery was introduced by Hazkins Kasunori Kashima in 1989. Till that time, any attempt to gain the airway resulted in scarring and restenosis of the airway and without much improvement in voice quality. Any attempt to increase the airway resulted in poor quality of voice. Kashima's surgery could change this dilemma and brought a safe airway without much loss of voice quality. Prophylactic tracheostomy was not done in our series. The only postoperative complication was formation of vocal process granuloma. It was found in three cases, all of whom were diabetic. They were managed by endoscopic removal.

Dyspnea index score was used to evaluate the effect of surgery in our study. It was very convenient and easy to administer and find out the efficacy of surgery.
Since it is the subjective measure of breathing quality, it reflects the true outcome and represents the improvement of quality-of-life. The follow-up questionnaire was given 6 weeks after the procedure. There was mild-to-moderate breathiness in the voice quality compared with preoperative voice. Maximum phonation time was assessed in all cases and the average was 8 seconds, which was fair enough for good voice. There was a case of cough on taking liquids after surgery. But, it was temporary and was managed with supraglottic swallowing technique. Apart from this single case, there was no swallowing problem.

All tracheostomized cases were decannulated successfully.

\section{CONCLUSION}

Endoscopic laser posterior cordotomy is an excellent surgery to improve the airway in bilateral abductor palsy. It does have only minimal detrimental effect on voice. Advantages of high success rate of decannualtion, short hospitalization, and cost-effectiveness make it a favorite treatment of choice in cases of established bilateral vocal cord palsy. To conclude, the endoscopic laser posterior cordotomy or Kashima's surgery is a very predictable surgery to reduce the severity of dyspnea in bilateral vocal cord palsy without greatly harming the voice. Swallowing ability too is hardly affected.

\section{REFERENCES}

1. Sulica L, Blitzer A. Vocal fold paralysis. In: Goding G, editor. Bilateral vocal fold immobility. Leipzig, Germany: SpringerVerlag; 2006. p. 237-246.

2. Baker $\mathrm{CH}$. Report of a case of abductor paralysis with removal of one vocal cord. J Mich Med Soc 1916 Oct;15:485-486.

3. Jackson C. Ventriculocordectomy, a new operation for the cure of goitrous glottis stenosis. Arch Surg 1922;4:257-274.

4. King BT. A new and function restoring operation for bilateral abductor cord paralysis. JAMA 1939;112(9):814-823.

5. Morrison LF. Further observations on the King operation for bilateral abductor paralysis. Ann Otol Rhinol Laryngol 1945;54:390-408.

6. Woodman D. A modification of the extralaryngeal approach to arytenoidectomy for bilateral abductor paralysis. Arch Otolaryngol 1946 Jan;43:63-65.

7. Thornell WC. Intralaryngeal approach for arytenoidectomy in bilateral abductor vocal cord paralysis. Arch Otolaryngol 1948 Apr;47(4):505-508.

8. Dennis DP, Kashima H. Carbon dioxide laser posterior cordectomy for treatment of bilateral vocal cord paralysis. Ann Otol Laryngol 1989 Dec;98(12 Pt 1):930-934.

9. Dursun G, Gökcan MK. Aerodynamic, aoustic and functional results of posterior transverse laser cordotomy for bilateral abductor vocal fold paralysis. J Laryngol Otol 2006 Apr;120(4): 282-288.

10. Proceedings of the International symposium on Care of the Professional voice and Phonomicrosurgery, Athens, Greece, September 1997. 